\title{
Adenovirus-mediated expression of bone morphogenetic protein-2 activates titanium particle-induced osteoclastogenesis and this effect occurs in spite of the suppression of TNF- $\alpha$ expression by siRNA
}

\author{
SHOUXUAN SUN ${ }^{1}$, HAOHUI GUO ${ }^{2}$, JIAN ZHANG $^{3}$, BO YU $^{2}$, KENING SUN $^{1}$ and QUNHUA JIN ${ }^{1}$ \\ ${ }^{1}$ Department of Orthopedics, Affiliated Hospital of Ningxia Medical University; ${ }^{2}$ Ningxia Medical University, \\ Yinchuan, Ningxia 750004; ${ }^{3}$ Institute of Pathology, Xi'an Jiaotong University, Xi'an, Shaanxi 710004, P.R. China
}

Received January 21, 2013; Accepted March 13, 2013

DOI: $10.3892 /$ ijmm.2013.1392

\begin{abstract}
The phagocytosis of wear particles by macrophages results in the secretion of pro-inflammatory cytokines, such as tumor necrosis factor- $\alpha(\mathrm{TNF}-\alpha)$, which play a major role in promoting osteoclast recruitment. The inhibition of TNF- $\alpha$ expression decreases osteoclastogenesis. In a previous study, we demonstrated that bone morphogenetic protein-2 (BMP-2) can activate wear debris-induced osteoclast recruitment in the presence of receptor activator of nuclear factor $(\mathrm{NF})-\kappa \mathrm{B}$ ligand (RANKL); however, whether these effects are associated with pro-inflammatory cytokines remains unclear. In this study, we constructed an adenoviral vector carrying TNF-small interfering RNA (siRNA) (Ad-TNF-siRNA), as well as a vector carrying both the BMP-2 gene and TNF- $\alpha$-siRNA (Ad-BMP-2-TNF-siRNA). The two adenoviral vectors significantly suppressed the expression of TNF- $\alpha$; however, only treatment with Ad-TNF-siRNA significantly inhibited osteoclastogenesis. We demonstrate that the overexpression of BMP-2, despite the suppression of TNF- $\alpha$ expression by Ad-BMP-2-TNF-siRNA, increases the size and number of titanium (Ti) particle-induced multinuclear osteoclasts, the expression of osteoclast genes, as well as the resorption area. There were no differences observed between Ti particle-induced and Ad-BMP-2-TNF-siRNA-induced osteoclast formation. Moreover, Ad-BMP-2-TNF-siRNA directly acted upon osteoclast precursors by increasing the level of c-Fos, regulating other signaling pathways, such as p38 phosphorylated c-Jun $\mathrm{N}$-terminal kinase (p-JNK) and phosphorylated I $\mathrm{KB}(\mathrm{p}-\mathrm{I} \kappa \mathrm{B})$. Taken together, these data demonstrate that treatment with Ad-BMP-2-TNF-siRNA increases wear debris-induced osteo-
\end{abstract}

Correspondence to: Dr Qunhua Jin, Department of Orthopedics, Affiliated Hospital of Ningxia Medical University, 804 Shengli Street, Yinchuan, Ningxia 750004, P.R. China

E-mail: ssxtian@sohu.com

Key words: adenovirus, bone morphogenetic protein-2, titanium particles, small interfering RNA, tumor necrosis factor- $\alpha$, osteoclast clast formation by activating c-Fos and that these effects are not associated with pro-inflammatory cytokines.

\section{Introduction}

The biological response to wear particles at the bone-implant interface is considered the main cause of aseptic loosening and osteolysis $(1,2)$, by increasing osteoclastogenesis and bone resorption (3). The phagocytosis of wear particles by macrophages results in the differentiation of macrophages into bone resorbing osteoclasts (4-6), as well as in the enhanced expression of pro-inflammatory cytokines, such as tumor necrosis factor- $\alpha$ (TNF- $\alpha$ ), interleukin (IL)-1 and IL-6 (7,8). TNF- $\alpha$ has been demonstrated to play a fundamental role in the pathogenesis of implant particle-induced osteolysis, which acts via receptors on osteoblastic cells to enhance receptor activator of nuclear factor (NF)- $\mathrm{B}$ ligand (RANKL) expression $(9,10)$. Moreover, previous studies have indicated that TNF- $\alpha$ directly stimulates macrophages to differentiate into osteoclasts by a mechanism independent of RANKL $(11,12)$. The knockdown of TNF- $\alpha$ expression has been shown to decrease osteoclast formation $(13,14)$.

Bone morphogenetic protein (BMP)-2 belongs to the superfamily of TGF- $\beta$ ligands, which initiate signaling by binding to BMP type I (BMPR-IA and BMPR-IB) and type II (BMPR-II) receptors, and then phosphorylate Smad, resulting in the translocation of Smad to the nucleus $(15,16)$. The significance of BMP-2 signaling in the osteoclast lineage has not yet been elucidated. A number of studies have suggested that BMPs can indirectly activate osteoclasts through the enhanced expression of osteoclastic-promoting factors by osteoblasts or stromal cells $(17,18)$ and that BMP-2 can directly stimulate osteoclast differentiation (19-21).

However, neither wear particles nor BMP-2 can induce osteoclast recruitment without RANKL, which is essential for osteoclastogenesis (22). It is well known that mitogen-activated protein (MAP) kinase family members [c-Jun N-terminal kinase (JNK), p38 and extracellular signal-regulated kinase $(\mathrm{ERK})], \mathrm{NF}-\kappa \mathrm{B}$ and activator protein-1 (AP-1) (c-Fos and c-Jun) are essential in osteoclastogenesis $(23,24)$; however, 
whether BMP-2 can affect these pathways remains unclear. In a previous study, it was demonstrated that BMP-2 increased the titanium (Ti) particle-mediated osteoclast differentiation of RAW264.7 cells in the presence of RANKL (25). However, whether TNF- $\alpha$, the major particle-induced inflammatory cytokine in the macrophage cell line, can alter the effects of BMP-2 expression remains unclear. In the present study, we constructed an adenoviral vector carrying TNF- $\alpha$-small interfering RNA (siRNA) (Ad-TNF- $\alpha$-siRNA), as well as a vector carrying both BMP-2 and TNF- $\alpha$ (Ad-BMP-2-TNF-siRNA). We hypothesized that siRNA targeting TNF- $\alpha$ can suppress the expression of TNF- $\alpha$. We also induced the overexpression BMP-2 to further characterize its role in Ti particle-induced osteoclastogensis.

\section{Materials and methods}

Cultured cells and reagents. RAW264.7 mouse macrophage cells (obtained from the Cell Bank of Chinese Academy of Sciences, Shanghai, China) were cultured in DMEM with glutaMAX (Gibco/Invitrogen, Carlsbad, CA, USA) containing $10 \%$ fetal bovine serum (FBS, HyClone, Logan, UT, USA) and $1 \%$ antibiotic-antimycotic (Gibco/Invitrogen) in a humidified atmosphere of $5 \% \mathrm{CO}_{2}$ at $37^{\circ} \mathrm{C}$.

Titanium particles. The Ti particles were kindly provided by Zimmer Inc., (Warsaw, IN, USA). The average particle size was $5 \mu \mathrm{m}$, and the particles were rinsed in ethanol 3 times and then rinsed 3 times with PBS. The particles were then autoclaved at $135^{\circ} \mathrm{C}$ for $15 \mathrm{~min}$, and mixed with culture medium at a concentration of $50 \mu \mathrm{g} / \mathrm{ml}$. The Ti suspensions were sonicated for $30 \mathrm{~min}$ prior to use.

Construction of adenoviral vectors expressing TNF-siRNA and $B M P-2-T N F-s i R N A$. To construct the adenovirus expressing TNF-siRNA (Ad-TNF-siRNA), siRNA targeting the TNF- $\alpha$ coding region (sense, 5'-GGUUGCCUCUGUCUCAGAATT-3'; and antisense, 5'-UUCUGAGACAGAGGCAACCTG-3') was firstly inserted into the KpnI and SpeI sites in the pSilencer 2.1-hU6 vector, then amplified with PCR and cloned into the pGEMT vector. The TNF- $\alpha$-siRNA was cut from the pGEMT vector with $K p n I$ and $S p e I$, and cloned into the same restriction sites of pAd5 E1-MCS-CMVeGFP. The plasmid was amplified by transformation into $\mathrm{DH} 5 \alpha$ cells and positive clones were selected and confirmed by DNA Miniprep and $K p n I$ digestion. The resultant plasmid, pAd5E1-hU6-TNF- $\alpha$ siRNA-CMVeGFP, was linearized with PacI digestion and subsequently co-transformed into HEK293 cells with E3 deleted Ad backbone.

To construct the adenovirus expressing BMP-2 and TNF- $\alpha$-siRNA (Ad-BMP-2-TNF- $\alpha$-siRNA), the full-length BMP-2 cDNA expressed in the pCDNA3.1 vector (KpnI and $\mathrm{XbaI}$ sites) was firstly cloned into the pAd5E3-CMV shuttle plasmid using the ClaI and SpeI sites. The plasmid was then amplified by transformation into DH5 $\alpha$ cells and positive clones (pAd5E3CMV-mBMP-2-pA) were selected and confirmed by DNA Miniprep and PacI digestion. To construct the pAd5E3-CMV-mBMP-2 backbone, pAd5E3-CMV-mBMP-2-PA was linearized with PacI digestion and subsequently co-transformed into BJ5183 cells with
pAd5-backbone using SwaI sites (26). Plasmids were amplified by transformation into DH5 $\alpha$ cells followed by DNA Maxiprep. To construct the adenoviral vector expressing both BMP-2 and TNF- $\alpha$-siRNA, pAd5E1-hU6 TNF- $\alpha$ siRNA-CMVeGFP was linearized with $P a c$ I digestion and subsequently co-transformed into HEK293 cells with linearized pAd5E3-CMV-mBMP-2 backbone.

Adenoviral vectors were purified on 3 rounds of cesium chloride $(\mathrm{CsCl})$ density gradients, and dialyzed with viral titers, determined by optical density and standard plaque assay as previously described (27).

Adenovirus infection of RAW264.7 cells. RAW264.7 cells were plated in a 24 -well plate at a density of $2 \times 10^{4}$ cells/well. Twenty-four hours after plating the cells, the cells were incubated at a multiplicity of infection (MOI) of 50 of either Ad-TNF-siRNA or Ad-BMP-2-TNF-siRNA in the presence of RANKL and Ti particles. After 4 days, total mRNA was extracted for gene expression by real-time RT-PCR and protein was extracted for western blot analysis or ELISA.

Cell proliferation assay. The cells were plated in 96-well plates at a density of $1 \times 10^{4}$ cells/well. Following treatment with RANKL (50 ng/ml; R\&D Systems, Minneapolis, MN, USA) and Ti particles, the cells were treated with a MOI of 50 of Ad-TNF-siRNA or Ad-BMP-2-TNF-siRNA. Cell viability was assessed by MTT assay (Sigma-Aldrich, St. Louis, MO, USA) according to the manufacturer's instructions.

Tartrate-resistant acid phosphatase-positive (TRAP) staining assay. In order to differentiate the RAW264.7 cells into osteoclasts, the cells were seeded in 96-well plates $\left(1 \times 10^{4}\right.$ cells/well) with RANKL $(50 \mathrm{ng} / \mathrm{ml})$ and Ti particles $(50 \mu \mathrm{g} / \mathrm{ml})$, and were subsequently treated with Ad-TNF-siRNA or Ad-BMP-2-TNF-siRNA. Multinucleated osteoclasts were observed on day 5. TRAP staining kits (Sigma-Aldrich) were used to evaluate TRAP expression. The TRAP(+) multinucleated cells that contained $\geq 3$ nuclei were counted as osteoclasts under an optical microscope. ImagePro Plus software was used to quantify the data.

Pit resorption assay. The effects of osteoclastic modulators were examined with Corning Osteo assay surface 24-well plates (Corning, Inc., Corning, NY, USA). RAW264.7 cells $(\sim 5,000$ cells/well) were transferred into each well for 9 days with a medium change on day 3 . After 9 days the plates were washed with $6 \%$ sodium hypochlorite solution to remove the cells. Individual pits or multiple pit clusters were observed using a microscope at x 5 magnification.

ELISA. To examine the TNF- $\alpha$ secretion, the RAW264.7 cells were plated in 96-well plates at a density of $10^{4}$ cells/well, and the cells were incubated for $6,12,24$ and $48 \mathrm{~h}$ with Ad-TNF-siRNA or Ad-BMP-2-TNF-siRNA in the presence of Ti particles $(50 \mu \mathrm{g} / \mathrm{ml})$. The control cells were only treated with Ti particles for the indicated periods of time. The levels of TNF- $\alpha$ secreted in the cell culture supernatant were measured using ELISA kits (Shanghai Lianshuo Biological Technology Co. Ltd., Shanghai, China) according to the manufacturer's instructions. 
Quantitative real-time PCR. Total RNA was isolated from the cells using TRIzol reagent (Invitrogen Life Technologies, Carlsbad, CA, USA) and quantified using NanoDrop 2000. RNA was reverse transcribed to cDNA using the RevertAid first strand cDNA synthesis kit (Fermentas, Beijing, China). Quantitative real-time PCR was performed in triplicate using the Bio-Rad iQ5 Gradient Real-Time PCR system (Bio-Rad, Hercules, CA, USA). All reactions were performed in a $25-\mu 1$ reaction volume containing $12.5 \mu \mathrm{l}$ of SYBR Premix Ex Taq $^{\mathrm{TM}}$ II (Takara Bio, Inc., Shiga, Japan) according to the manufacturer's instructions. The sequences of the primers used were: TRAP (forward), 5'-GCAGCCAAGGAGGACTAC and (reverse), 5'-CCCACTCAGCACATAGCC; nuclear factor of activated T-cells, cytoplasmic, calcineurin-dependent 1 (NFATc1) (forward), 5'-TCTTCCGAGTTCCATCC and (reverse), 5'-ACAGCACCATCTTCTTCC; dendrocyte expressed seven transmembrane protein (DC-STAMP) (forward), 5'-TGTGGCTGGAAGTTGAGAATG and (reverse), 5'-AGATAGGTCAGGACAGGAAGG; cathepsin K (CTSK) (forward), 5'-CAGAACGGAGGCATTGAC and (reverse), 5'-CGATGGACACAGAGATGG; TNF- $\alpha$ (forward), 5'-TCTTCTCATTCTGCTTGTG and (reverse), 5'-ACTTGG TGGTTTCTACG; BMP-2 (forward), 5'-TTGTATGTGGA CTCAGTGATGTG and (reverse), 5'-AGTTCAGGTGG TCAGCAAGG; and GAPDH (forward), 5'-TCAACGGC ACAGTCAAGG and (reverse), 5'-ACTCCACGACATAC TCAGC.

Western blot analysis. Whole cells were extracted in RIPA buffer containing protease and phosphatase inhibitors (Sigma-Aldrich). Proteins were resolved by SDS-PAGE, transferred onto PVDF membranes (Millipore, Billerica, MA, USA) and incubated overnight at $4^{\circ} \mathrm{C}$ with primary antibodies against BMP-2 (Abcam, Cambridge, UK), c-Fos (Cell Signaling Technology, Inc., Danvers, MA, USA), phosphorylated p38 (p-p38) (Cell Signaling Technology, Inc.), phosphorylated JNK (p-JNK) (Anbo Biotechnology Co., Ltd., San Francisco, CA, USA) and phosphorylated $\mathrm{I} \kappa \mathrm{B}(\mathrm{p}-\mathrm{I} \kappa \mathrm{B})$ (Anbo Biotechnology Co., Ltd.). The same membranes were probed with anti- $\beta$-actin antibodies (Sigma-Aldrich) to normalize protein loading in each lane. Immunoreactive bands were visualized using ECL Plus substrate (GE Healthcare Life Sciences, Shanghai, China).

Statistical analysis. All experiments were performed in triplicate. Significant differences were determined using one-way ANOVA. A p-value $<0.05$ was considered to indicate a statistically significant difference.

\section{Results}

Adenoviral vectors expressing TNF-siRNA and BMP-2$T N F$-siRNA in RAW264.7 cells. We constructed the adenoviral vectors expressing BMP-2-TNF-siRNA (Ad-BMP-2-TNFsiRNA) and TNF-siRNA (Ad-TNF-siRNA; Fig. 1A). First we confirmed that the BMP-2 mRNA expression increased in the RAW264.7 cells and that the MOI of 50 of Ad-BMP-2-TNFsiRNA induced the optimal expression level of BMP-2 compared to the control (Fig. 1C). This result was confirmed by western blot analysis (Fig. 1B). We then confirmed the inhibitory effect of the 2 vectors on the expression of TNF- $\alpha$. As shown in Fig. 1D, the Ad-TNF-siRNA and Ad-BMP-2-TNF-siRNA vectors significantly decreased the TNF- $\alpha$ levels at a MOI of 50 and 70. ELISA analysis of the cell supernatants further demonstrated that although Ti particles markedly induced the expression of TNF- $\alpha$, this expression was successfully inhibited by Ad-TNF-siRNA and Ad-BMP-2-TNF-siRNA at a MOI of 50 (Fig. 1E). Furthermore, there was no statistically significant difference observed between Ad-TNF-siRNA and Ad-BMP-2-TNF-siRNA in inhibiting the expression of TNF- $\alpha$. These results indicated that we successfully constructed the vectors, Ad-TNF-siRNA and Ad-BMP-2-TNF-siRNA. At a MOI of 50, the 2 vectors inhibited particle-induced TNF- $\alpha$ expression; Ad-BMP-2-TNF-siRNA also induced the optimal expression level of BMP-2.

Ad-BMP-2-TNF-siRNA synergizes with Ti particles in enhancing the differentiation of osteoclast precursors. In a previous study, it was demonstrated that BMP-2 increased the Ti particle- and RANKL-induced osteoclast differentiation of RAW264.7 cells (25). To confirm whether BMP-2 can effect osteoclast formation independently of the expression status of TNF- $\alpha$, RAW264.7 cells were pre-treated with RANKL $(50 \mathrm{ng} / \mathrm{ml})$ and Ti particles $(50 \mu \mathrm{g} / \mathrm{ml})$ for $1 \mathrm{~h}$ and subsequently treated with Ad-TNF-siRNA or Ad-BMP-2-TNF-siRNA for 6 days. We performed TRAP staining to visualize the differentiated osteoclasts (Fig. 2A). Although none of these treatments induced osteoclast formation without RANKL (data not shown), a high number of TRAP-positive cells was observed following treatment with Ti particles in the presence of RANKL, and we also observed that Ad-TNF-siRNA significantly inhibited this effect. Only small, mononucleated cells were observed. However, when the RAW264.7 cells were treated with Ad-BMP-2-TNF-siRNA, large, multinucleated cells were observed again. These results are presented in Fig. 2B and $\mathrm{C}$; following treatment with Ad-BMP-2-TNF-siRNA, the average number of osteoclasts per well and the average size of the 20 largest osteoclasts per well was increased by least 3-fold compared to the Ad-TNF-siRNA-treated group, and there were no statistically significant differences observed between the Ti particle- and Ad-BMP-2-TNF-siRNA-treated groups. The pit resorption per well further confirmed these results, showing that the resorption area significantly increased in both the Ti particle- and Ad-BMP-2-TNF-siRNA-treated group (Fig. 2D). By contrast, treatment with Ad-TNF-siRNA significantly decreased the resorption area. These data indicate that BMP-2 enhances Ti particle-mediated osteoclast formation in the presence of RANKL, and that this effect occurs independently of TNF- $\alpha$ expression.

Adenoviral vectors do not affect proliferation of RAW264.7 cells. To determine whether Ad-TNF-siRNA or Ad-BMP2-TNF-siRNA enhances osteoclast formation by increased proliferation, we used MTT assay (Fig. 3). We detected only a slight difference between treatment with Ad-TNF-siRNA, Ad-BMP-2-TNF-siRNA and the control. Therefore, it can be concluded that treatment with Ad-TNF-siRNA and Ad-BMP-2TNF-siRNA does not affect RAW264.7 cell proliferation.

BMP-2 increases the Ti particle-mediated expression of osteoclast genes. We used real-time PCR to examine the 
A

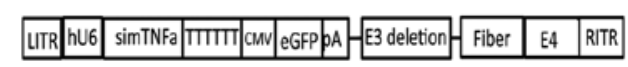

Ad-TNF- $\alpha-$-siRNA

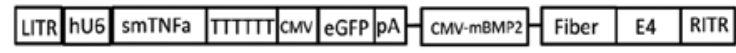

Ad-BMP-2-TNF- $\alpha$-siRNA
B

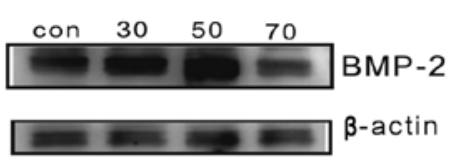

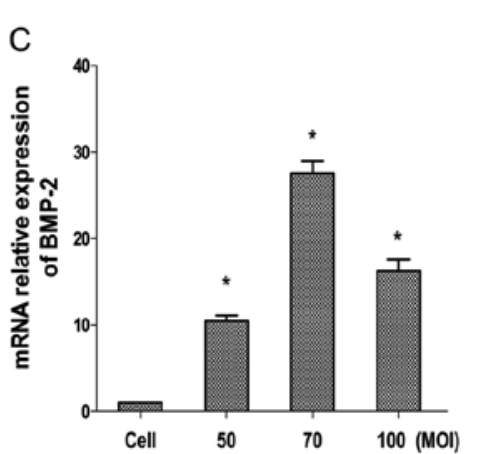
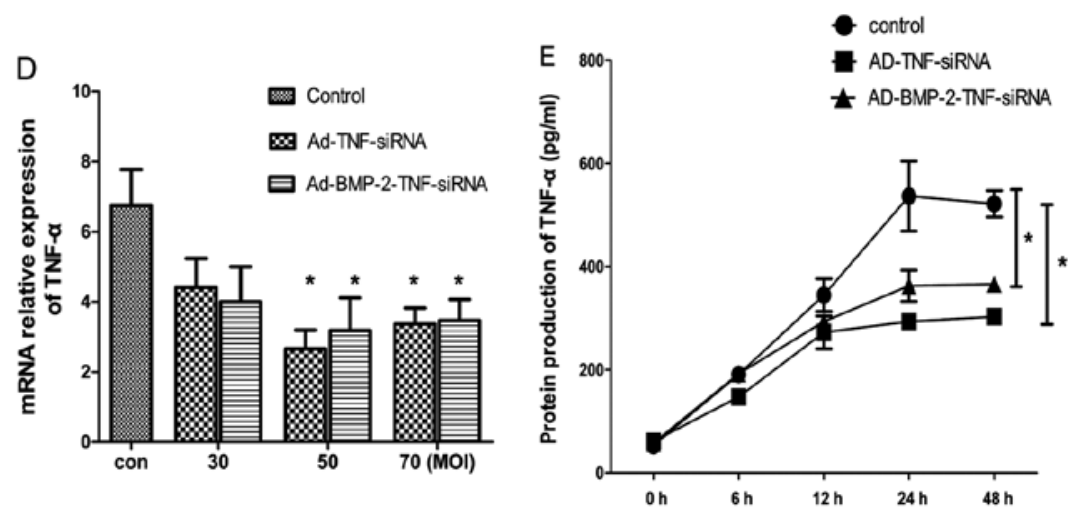

Figure 1. Adenoviral vector expressing TNF-siRNA and BMP-2-TNF-siRNA. (A) The construction of adenovirus vectors expressing TNF-siRNA (Ad-TNF-siRNA) and BMP-2-TNF-siRNA (Ad-BMP-2-TNF-siRNA). (B) The RAW264.7 cells were harvested following treatment with Ad-BMP-2-TNFsiRNA for $24 \mathrm{~h}$, then bone morphogenetic protein-2 (BMP-2) expression was detected by western blot analysis. (C) Quantitative real-time PCR analysis of BMP-2 expression at increasing multiplicities of infection (MOIs). (D) The RAW264.7 cells were pre-treated with titanium (Ti) particles $(50 \mu \mathrm{g} / \mathrm{ml})$ for $1 \mathrm{~h}$ and subsequently treated with Ad-TNF-siRNA or Ad-BMP-2-TNF-siRNA for $24 \mathrm{~h}$ at a MOI of 30,50 and 70. Quantitative real-time PCR was used to detect the expression of TNF- $\alpha$ in the treated cells, compared to that in the control cells pre-treated with Ti particles for $24 \mathrm{~h}$. (E) ELISA was used to detect the protein levels of TNF- $\alpha$ in the RAW264.7 cells transfected with Ad-TNF-siRNA (MOI of 50) or Ad-BMP-2-TNF-siRNA (MOI of 50) at the indicated times following treatment with Ti particles $(50 \mu \mathrm{g} / \mathrm{ml})$ for $1 \mathrm{~h} .{ }^{*} \mathrm{p}<0.01$.
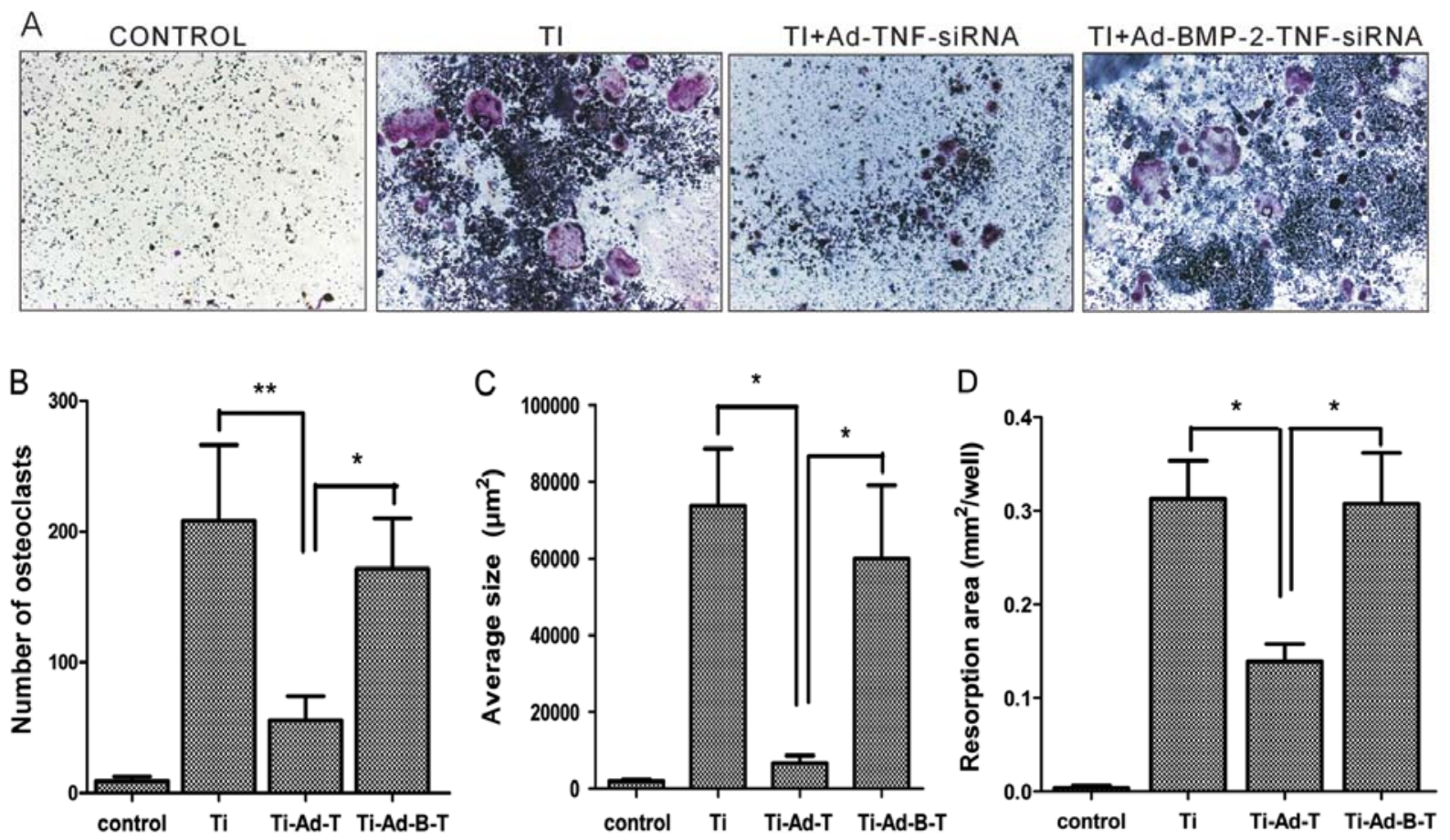

Figure 2. Effects of treatment with Ad-TNF-siRNA (Ad-T) and Ad-BMP-2-TNF-siRNA (Ad-B-T) on titanium (Ti) particle-mediated osteoclast formation. (A) Tartrate-resistant acid phosphatase-positive (TRAP) staining of RAW264.7 cell-derived osteoclasts. RAW264.7 cells (control) were pre-treated with receptor activator of NF- $\mathrm{kB}$ ligand (RANKL) $(50 \mu \mathrm{g} / \mathrm{ml})$ and Ti particles $(50 \mu \mathrm{g} / \mathrm{ml})$ for $1 \mathrm{~h}$ (Ti group) and subsequently treated with or without Ad-TNF-siRNA or Ad-BMP-2-TNF-siRNA for 6 days. (B) Quantitative analysis was used to detect the number of osteoclasts per well. (C) The average size of the 10 largest osteoclasts in each well. (D) The pit resorption area per well. ${ }^{*} \mathrm{P}<0.05 ;{ }^{* *} \mathrm{P}<0.01$.

expression of the osteoclast marker genes, NFAT-c1, TRAP, DC-STAMP and CTSK (28-31), to further determine the effect of Ad-BMP-2-TNF-siRNA on osteoclast formation (Fig. 4).
PCR analysis indicated that treatment with Ti particles and Ad-BMP-2-TNF-siRNA exerted similar effects on TRAP, NAFTc1, DC-STAMP and CTSK gene expression. The 


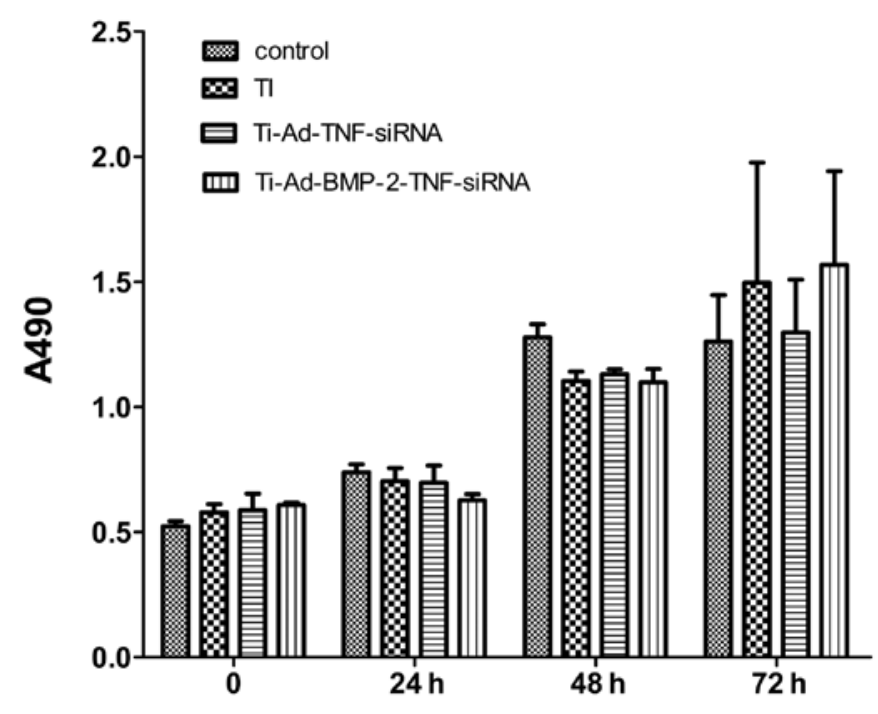

Figure 3. Adenoviral vectors do not affect RAW264.7 cell proliferation. RAW264.7 cells (control) were pre-treated with receptor activator of NF- $\kappa$ B ligand $(\mathrm{RANKL})(50 \mu \mathrm{g} / \mathrm{ml})$ and titanium $(\mathrm{Ti})$ particles $(50 \mu \mathrm{g} / \mathrm{ml})$ for $1 \mathrm{~h}$ and subsequently treated with or without Ad-TNF-siRNA or Ad-BMP-2-TNF-siRNA for the indicated periods of time. The cell proliferation was expressed as the absorbance ratio.

expression of TRAP, NAFTc1 and CTSK genes was significantly increased; however, the increase in the expression of the DC-STAMP gene was not statistically significant.

Ad-BMP-2-TNF-siRNA synergizes with Ti particles in c-Fos expression. c-Fos is expressed during the early stages of osteoclast formation and is essential for RANKL-mediated osteoclast differentiation (32). Thus, we observed its expression from day 1 to 4 following treatment with Ad-TNF-siRNA and Ad-BMP-2-TNF-siRNA. Immunoblot analysis showed that the expression of c-Fos was effectively inhibited by Ad-TNF-siRNA; only a weak c-Fos expression was observed. However, following treatment with Ad-BMP-2-TNF-siRNA, a higher c-Fos expression was observed from day 1 to 4 (Fig. 5). To further investigate the signaling pathways which may be activated by BMP-2, we detected the expression of p-p38, p-IкB and p-JNK by western blot analysis (Fig. 6). When the cells were incubated with Ad-BMP-2-TNF-siRNA for 10 to $120 \mathrm{~min}$, the expression level of p-p38, p-IкB and p-JNK was higher compared to the cells treated with Ad-TNF-siRNA. These results suggested that BMP-2 synergized with Ti particles during osteoclastogenesis by directly stiumulating the expression of p-IкB p-JNK and p-p38.

\section{Discussion}

Increasing osteoclastogenesis and the inhibition of bone formation is considered the main cause of aseptic loosening and osteolysis; thus, it is important to find a balance between bone formation and resorption. Previous studies have mainly focused on the inhibition of osteoclast formation $(33,34)$, and the significance of increasing bone formation has received little attention. However, the role of BMP-2 in promoting osteoblast differentiation has been extensively investigated $(35,36)$. It has been approved by the FDA as an alternative to bone grafts, for spinal fusion, long bone fractures and healing in a number of orthopedic and maxillofacial applications $(37,38)$. However, its function in particle-induced aseptic loosening and osteolysis has not yet been elucidated. In this study, we found that inducing
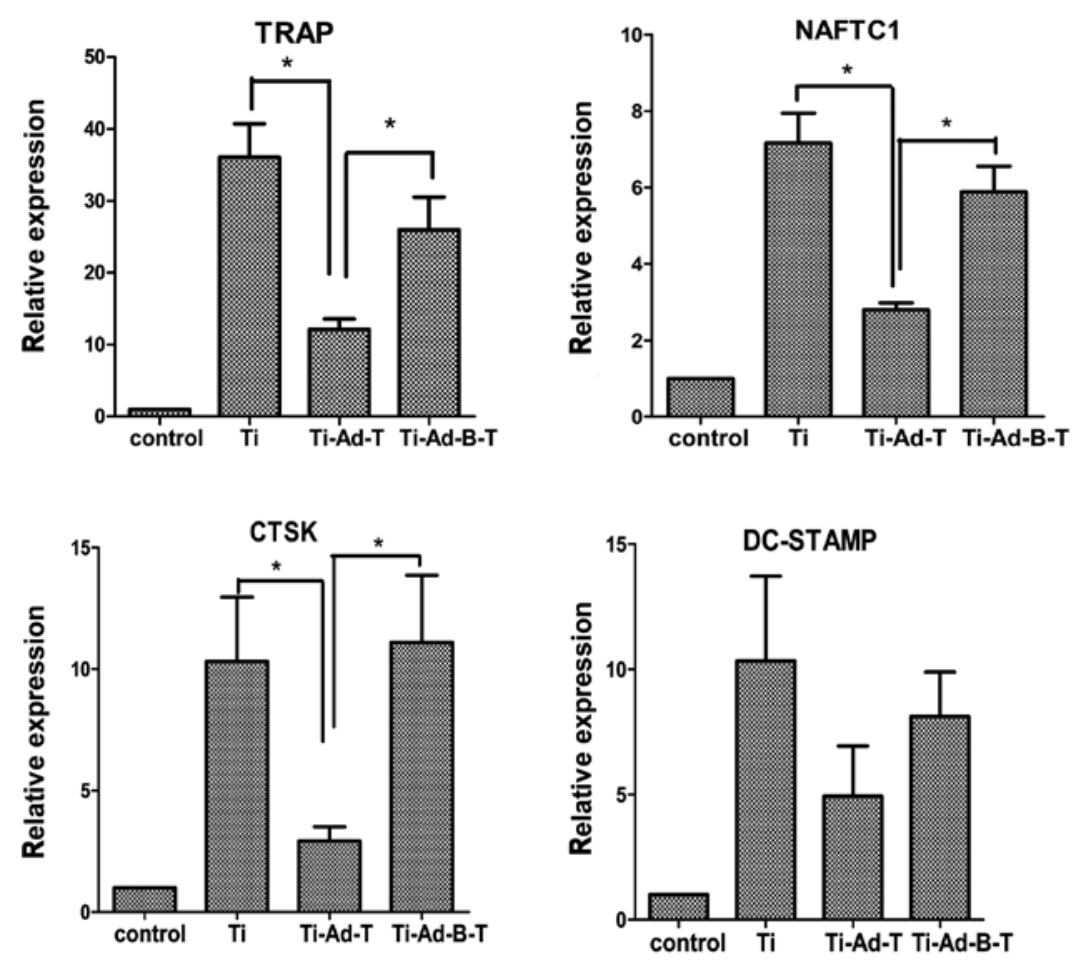

Figure 4. Expression of osteoclast marker genes. The RAW264.7 cells were pre-treated with receptor activator of NF- $\mathrm{kB}$ ligand (RANKL) $(50 \mu \mathrm{g} / \mathrm{ml})$ and titanium (Ti) particles $(50 \mu \mathrm{g} / \mathrm{ml})$ for $1 \mathrm{~h}$ and subsequently treated with or without Ad-TNF-siRNA (Ad-T) or Ad-BMP-2-TNF-siRNA (Ad-B-T) for 6 days. * $<<0.05$. 


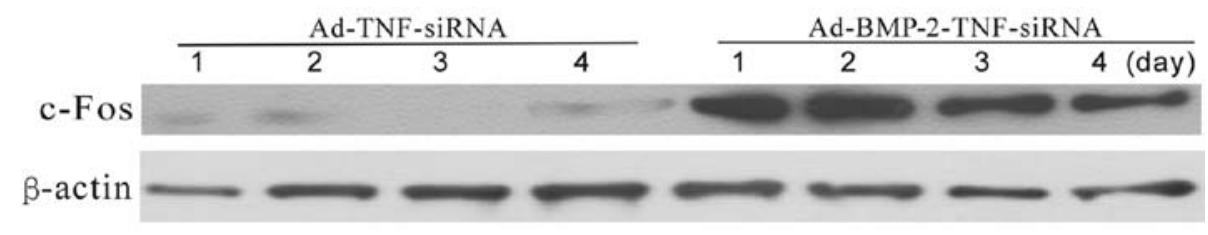

Figure 5. Western blot analysis was used to detect the expression of c-Fos. RAW264.7 cells were pre-treated with receptor activator of NF-kB ligand (RANKL) $(50 \mu \mathrm{g} / \mathrm{ml})$ and titanium (Ti) particles $(50 \mu \mathrm{g} / \mathrm{ml})$ for $1 \mathrm{~h}$ and subsequently treated with Ad-TNF-siRNA or Ad-BMP-2-TNF-siRNA [multiplicity of infection (MOI) of 50] for the indicated periods of time.

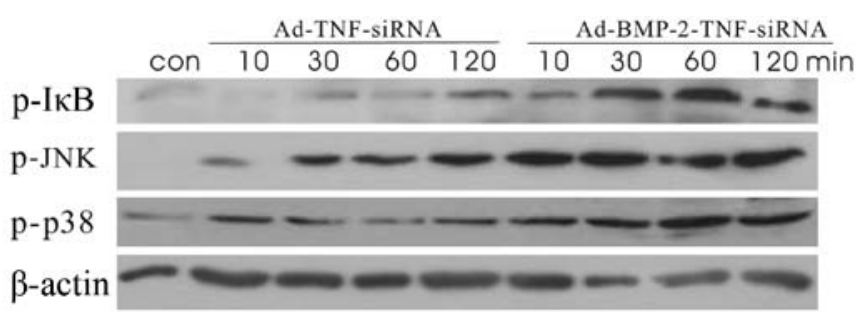

Figure 6. Bone morphogenetic protein-2 (BMP-2) regulates the expression of p-p38, p-JNK, p-IкB. RAW264.7 cells were pre-treated with receptor activator of NF- $\kappa B$ ligand (RANKL) $(50 \mu \mathrm{g} / \mathrm{ml})$ and titanium (Ti) particles $(50 \mu \mathrm{g} / \mathrm{ml})$ for $1 \mathrm{~h}$ and subsequently treated with Ad-TNF-siRNA or Ad-BMP-2-TNF-siRNA [multiplicity of infection (MOI) of 50] for the indicated periods of time. p-p38, $\mathrm{p}$-IкB and $\mathrm{p}-\mathrm{JNK}$ expression was detected by western blot analysis.

the overexpression of BMP-2 by adenoviral vectors increased multinuclear osteoclast formation with suboptimal levels of RANKL; this effect occurred even though we effectively inhibited the expression of TNF- $\alpha$. Our study challenges the view that BMP-2 may be used in the treatment of osteolysis (39-41).

The process of particle-induced osteolysis is complex and involves the interaction of numerous cells, cytokines, chemokines and growth factors (42). TNF- $\alpha$ has been demonstrated to play a fundamental role in the pathogenesis of implant particle-induced osteolysis. It has been reported that osteoclast precursors are direct targets of TNF- $\alpha(13,43)$. Our study confirmed this result; we constructed an adenoviral vector carrying TNF-siRNA which significantly inhibited Ti particleinduced osteoclast formation. In a previous study, it was shown that BMP-2 increased the Ti particle- and RANKL-induced osteoclast differentiation of RAW264.7 cells (25). In order to determine the crosstalk between BMP- 2 and TNF- $\alpha$, in this study, we constructed another adenoviral vector carrying both BMP-2 and TNF-siRNA (Ad-BMP-2-TNF-siRNA). We found that treatment with Ad-BMP-2-TNF-siRNA increased the size and number of multinuclear osteoclasts, the expression of osteoclast genes, as well as the resorption area, compared to treatment with Ad-TNF-siRNA, which significantly suppressed osteoclast formation. We also demonstrated that there were no significant differences between Ti particle-induced and Ad-BMP-2-TNF-siRNA-induced osteoclast formation. There was no significant difference in cell viability between the groups. This result indicated that BMP-2 directly acts upon osteoclast precursors to stimulate osteoclast formation, and that this effect is not associated with pro-inflammatory cytokines.

It has been demonstrated that c-Fos and NFATc1 play a critical role in the regulation of genes for osteoclast differentiation. These two transcription factors are also functionally linked together; c-Fos is essential for the RANKL-mediated induction of NFATc1 and it further regulates NFATc1 gene expression by binding to the promoter region of NFATc1 (44). In this study, we demonstrate that treatment with Ad-BMP-2TNF-siRNA stimulates c-Fos and NFATc1 expression, and acts upon downstream signaling pathways, such as p-JNK p-p38 and p-IкB; this effect occurs in spite of the inhibition of these pathways by treatment with TNF- $\alpha$-siRNA.

In conclusion, we demonstrate that BMP-2 increases Ti particle-induced osteoclast differentiation of RAW264.7 cells in the presence of suboptimal levels of RANKL by activating the $\mathrm{p}-\mathrm{JNK}$ p-p38 and p-IкB signaling pathways.

\section{Acknowledgements}

We thank Miss Hua Guan and Jixiao Li for their excellent technical assistance. This study was supported by grants from the National Natural Science Foundation of China (NSFC grant no. 81060146).

\section{References}

1. Jacobs JJ, Roebuck KA, Archibeck M, Hallab NJ and Glant TT: Osteolysis: basic science. Clin Orthop Rel Res 393: 71-77, 2001.

2. Ingham E and Fisher J: The role of macrophages in osteolysis of total joint replacement. Biomaterials 26: 1271-1286, 2005.

3. Haynes DR, Crotti TN, Potter AE, et al: The osteoclastogenic molecules RANKL and RANK are associated with periprosthetic osteolysis. J Bone Joint Surg Br 83: 902-911, 2001.

4. Kobayashi A, Freeman MA, Bonfield W, et al: Number of polyethylene particles and osteolysis in total joint replacements. A quantitative study using a tissue-digestion method. J Bone Joint Surg Br 79: 844-848, 1997.

5. Sabokbar A, Fujikawa Y, Neale S, Murray DW and Athanasou NA: Human arthroplasty derived macrophages differentiate into osteoclastic bone resorbing cells. Ann Rheum Dis 56: 414-420, 1997.

6. Sabokbar A, Pandey R and Athanasou NA: The effect of particle size and electrical charge on macrophage-osteoclast differentiation and bone resorption. J Mater Sci Mater Med 14: 731-738, 2003.

7. González O, Smith RL and Goodman SB: Effect of size, concentration, surface area, and volume of polymethylmethacrylate particles on human macrophages in vitro. J Biomed Mater Res 30: 463-473, 1996.

8. O'Keefe RJ, Rosier RN, Teot LA, Stewart JM and Hicks DG: Cytokine and matrix metalloproteinase expression in pigmented villonodular synovitis may mediate bone and cartilage destruction. Iowa Orthop J 18: 26-34, 1998.

9. Xu JW, Konttinen YT, Lassus J, Natah S, Ceponis A and Solovieva S: Tumor necrosis factor-alpha (TNF-alpha) in loosening of total hip replacement (THR). Clin Exp Rheumatol 14: 643-648, 1996.

10. Ross FP: RANKing the importance of measles virus in Paget's disease. J Clin Invest 105: 555-558, 2000.

11. Kobayashi K, Takahashi N, Jimi E, et al: Tumor necrosis factor- $\alpha$ stimulates osteoclast differentiation by a mechanism independent of the ODF/RANKLRANK interaction. J Exp Med 191: 275-286, 2000 
12. Azuma Y, Kaji K, Katogi R, Takeshita S and Kudo A: TNF-o induces differentiation of and bone resorption by osteoclasts. J Biol Chem 275: 4858-4864, 2000.

13. Qin CQ, Ding Y, Huang DS, Xu J, Ma RF and Huang JB: Downregulation of TNF-alpha by small interfering RNA inhibits particle-induced Inflammation in vitro. Artif Organs 35: 706-714, 2000.

14. Howard KA, Paludan SR, Behlke MA, Besenbacher F, Deleuran B and Kjems J: Chitosan/siRNA nanoparticle-mediated TNF-alpha knockdown in peritoneal macrophages for anti-inflammatory treatment in a murine arthritis model. Mol Ther 17: 162-168, 2009.

15. Suzuki A, Thies RS, Yamaji N, Song JJ, Wozney JM and Murakami K: A truncated bone morphogenetic protein receptor affects dorsal-ventral patterning in the early Xenopus embryo. Proc Natl Acad Sci USA 91: 10255-10259, 1994.

16. Yamaji N, Celeste AJ, Thies RS, et al: A mammalian serine/threonine kinase receptor specifically binds BMP-2 and BMP-4. Biochem Biophys Res Commun 205: 1944-1951, 1994

17. Abe E, Yamamoto M, Taguchi Y, et al: Essential requirement of BMPs-2/4 for both osteoblast and osteoclast formation in murine bone marrow cultures from adult mice: antagonism by noggin J Bone Miner Res 15: 663-673, 2000.

18. Wutzl A, Brozek W, Lernbass I, et al: Bone morphogenetic proteins 5 and 6 stimulate osteoclast generation. J Biomed Mater Res A 77: 75-83, 2006.

19. Jensen ED, Pham L, Billington CJ Jr, et al: Bone morphogenic protein 2 directly enhances differentiation of murine osteoclast precursors. J Cell Biochem 109: 672-682, 2010.

20. Giannoudis PV, Kanakaris NK and Einhorn TA: Interaction of bone morphogenetic proteins with cells of the osteoclast lineage: review of the existing evidence. Osteoporos Int 18: 1565-1581, 2007.

21. Falany ML, Thames AM III, McDonald JM, et al: Osteoclasts secrete the chemotactic cytokine mim-1. Biochem Biophys Res Commun 281: 180-185, 2001.

22. Roodman GD: Regulation of osteoclast differentiation. Ann NY Acad Sci 1068: 100-109, 2006.

23. Abbas S, Clohisy JC and Abu Amer Y: Mitogen-activated protein (MAP) kinases mediate PMMA-induction of osteoclasts. J Orthop Res 21: 1041-1048, 2003.

24. AE Grigoriadis, ZQ Wang, MG Cecchini, et al: c-Fos: a key regulator of osteoclast-macrophage lineage determination and bone remodeling. Science 266: 443-448, 1994.

25. Zheng Y, Wang L, Zhang X, et al: BMP2/7 heterodimer can modulate all cellular events of the in vitro RANKL-mediated osteoclastogenesis, respectively, in different dose patterns. Tissue Eng Part A 18:621-630, 2012

26. Anderson RD, Haskell RE, Xia H: A simple method for the rapid generation of recombinant adenovirus vectors. Gene Ther 7 : 1034-1038, 2000.

27. Palmer GD, Gouze E, Gouze JN, Betz OB, Evans CH and Ghivizzani SC: Gene transfer to articular chondrocytes with recombinant adenovirus. Methods Mol Biol 215: 235-246, 2003.

28. Takayanagi H, Kim S, Koga T, et al: Induction and activation of the transcription factor NFATc1 (NFAT2) integrate RANKL signaling in terminal differentiation of osteoclasts. Dev Cell 3 : 889-901, 2002 .
29. Novack DV and Teitelbaum SL: The osteoclast: friend or foe? Annu Rev Pathol 3: 457-484, 2008.

30. Yagi M, Miyamoto T, Sawatani Y, et al: DC-STAMP is essential for cell-cell fusion in osteoclasts and foreign body giant cells. J Exp Med 202: 345-351, 2005.

31. Hsu H, Lacey DL, Dunstan CR, et al: Tumor necrosis factor receptor family member RANK mediates osteoclast differentiation and activation induced by osteoprotegerin ligand. Proc Natl Acad Sci USA 96: 3540-3545, 1999.

32. Arai A, Mizoguchi T, Harada S, et al: Fos plays an essential role in the upregulation of RANK expression in osteoclast precursors within the bone microenvironment. J Cell Sci 125: 2910-2917, 2012.

33. Abu-Amer Y, Darwech I and Clohisy JC: Aseptic loosening of total joint replacements: mechanisms underlying osteolysis and potential therapies. Arthritis Res Ther 9 (Suppl 1): S6, 2007.

34. Shen Z, Crotti TN, McHugh KP, et al: The role played by cell-substrate interactions in the pathogenesis of osteoclast-mediated peri-implant osteolysis. Arthritis Res Ther 8: R70, 2006

35. Cao $X$ and Chen D: The BMP signaling and in vivo bone formation. Gene 357: 1-8, 2005.

36. Bessa PC, Casal M and Reis RL: Bone morphogenetic proteins in tissue engineering: the road from laboratory to clinic, part II (BMP delivery). J Tissue Eng Regen Med 2: 81-96, 2008.

37. Gautschi OP, Frey SP and Zellweger R: Bone morphogenetic proteins in clinical applications. ANZ J Surg 77: 626-631, 2007.

38. Kirker-Head CA, Boudrieau RJ and Kraus KH: Use of bone morphogenetic proteins for augmentation of bone regeneration. J Am Vet Med Assoc 231: 1039-1055, 2007.

39. Li G, Li Q, Lin LJ, Duan X and Zhang XQ: Slow-release recombinant human bone morphogenetic protein-2 suppresses chromium wear particle-induced osteolysis in rats. Nan Fang Yi Ke Da Xue Xue Bao 32: 391-395, 2012 (In Chinese).

40. Helgeson MD, Lehman RA Jr, Patzkowski JC, Dmitriev AE, Rosner MK and Mack AW: Adjacent vertebral body osteolysis with bone morphogenetic protein use in transforaminal lumbar interbody fusion. Spine J 11: 507-510, 2012.

41. Southwood LL, Frisbie DD, Kawcak CE, Ghivizzani SC, Evans $\mathrm{CH}$ and McIlwraith CW: Evaluation of Ad-BMP-2 for enhancing fracture healing in an infected defect fracture rabbit model. J Orthop Res 22: 66-72, 2004.

42. Beraudi A, Stea S, Cremonini S, Visentin M and Toni A: Assessment of five interleukins in human synovial fluid as possible markers for aseptic loosening of hip arthroplasty. Artif Organs 33: 538-543, 2009.

43. Lam J, Takeshita S, Barker JE, Kanagawa O, Ross FP and Teitelbaum SL: TNF-alpha induces osteoclastogenesis by direct stimulation of macrophages exposed to permissive levels of RANK ligand. J Clin Invest 106: 1481-1488, 2000.

44. Huang H, Chang EJ, Ryu J, Lee ZH, Lee Y and Kim HH: Induction of c-Fos and NFATc1 during RANKL-stimulated osteoclast differentiation is mediated by the p38 signaling pathway. Biochem Biophys Res Commun 351: 99-105, 2006. 